\title{
Cholangiocytes derived from human induced pluripotent stem cells for disease modeling and drug validation
}

\author{
Fotios Sampaziotis $^{1,2,7}$, Miguel Cardoso de Brito ${ }^{1,12}$, Pedro Madrigal ${ }^{1,3,12}$, Alessandro Bertero ${ }^{1}$, \\ Kourosh Saeb-Parsy ${ }^{2}$, Filipa A C Soares ${ }^{1}$, Elisabeth Schrumpf ${ }^{4-6}$, Espen Melum ${ }^{4,5}$, Tom H Karlsen ${ }^{4-6}$, \\ J Andrew Bradley $^{2}$, William T H Gelson ${ }^{7}$, Susan Davies ${ }^{8}$, Alastair Baker ${ }^{9}$, Arthur Kaser ${ }^{10}$, Graeme J Alexander ${ }^{11}$, \\ Nicholas R F Hannan ${ }^{1,13}$ \& Ludovic Vallier ${ }^{1,3,13}$
}

\begin{abstract}
The study of biliary disease has been constrained by a lack of primary human cholangiocytes. Here we present an efficient, serum-free protocol for directed differentiation of human induced pluripotent stem cells into cholangiocyte-like cells (CLCs). CLCs show functional characteristics of cholangiocytes, including bile acids transfer, alkaline phosphatase activity, $\gamma$-glutamyltranspeptidase activity and physiological responses to secretin, somatostatin and vascular endothelial growth factor. We use CLCs to model in vitro key features of Alagille syndrome, polycystic liver disease and cystic fibrosis (CF)-associated cholangiopathy. Furthermore, we use CLCs generated from healthy individuals and patients with polycystic liver disease to reproduce the effects of the drugs verapamil and octreotide, and we show that the experimental CF drug VX809 rescues the disease phenotype of CF cholangiopathy in vitro. Our differentiation protocol will facilitate the study of biological mechanisms controlling biliary development, as well as disease modeling and drug screening.
\end{abstract}

Cholangiocytes are the main site of dysfunction in cholangiopathies, a diverse group of bile duct disorders that includes inherited diseases such as CF-associated cholangiopathy, developmental diseases such as Alagille syndrome and autoimmune diseases such as primary biliary cirrhosis, as well as drug- and toxin-induced conditions ${ }^{1}$. Cholangiopathies carry significant morbidity and mortality, accounting for up to a third of adult and more than $70 \%$ of pediatric liver transplantations ${ }^{2}$. However, research on their pathophysiology has been limited by poor access to primary biliary tissue, difficulties in culturing primary cholangiocytes in vitro and inadequate animal disease models $^{3}$.

The capacity of human induced pluripotent stem cells (hiPSCs) to proliferate indefinitely in culture and differentiate into a broad spectrum of cell types makes them well suited to in vitro disease modeling $^{4,5}$. Early methods for deriving cholangiocytes from hiPSCs ${ }^{6}$ were based on spontaneous differentiation and had limited characterization of the resulting cells ${ }^{7,8}$. Despite recent advances toward guided differentiation of hiPSCs to CLCs ${ }^{9}$, existing protocols show poor differentiation efficiency $(<31 \%)$, and the derived cells differ considerably from primary biliary tissue in their transcriptional profiles. Furthermore, in vitro-generated cholangiocytes have not been shown to reproduce key functions of bona fide cholangiocytes, such as enzymatic activity (for example, alkaline phosphatase (ALP) and $\gamma$-glutamyl transferase (GGT)), responses to hormonal stimuli (secretin and somatostatin) and chloride transfer through cystic fibrosis transmembrane regulator (CFTR) activity ${ }^{7-9}$. Demonstration of these properties is essential for recapitulating cholangiopathies and studying the effects of therapeutic agents. Finally, current systems diverge from the physiological pathways controlling biliary development in $v i v o^{7-9}$, limiting their value for developmental studies.

Here we report a stepwise method for cholangiocyte differentiation that recapitulates native biliary development (Fig. 1a). The quality, functionality and purity of the resulting CLCs are substantially higher than those of cells generated by previous methods (see Supplementary Note, Supplementary Methods and Supplementary Fig. 1 for detailed comparison).

\section{RESULTS}

Cholangiocyte progenitors generated from hiPSCs

We focused first on the generation of bipotent hepatoblasts, the common progenitor of hepatocytes and cholangiocytes ${ }^{10}$. To achieve this goal,

\footnotetext{
${ }^{1}$ Wellcome Trust-Medical Research Council Stem Cell Institute, Anne McLaren Laboratory, Department of Surgery, University of Cambridge, Cambridge, UK. ${ }^{2}$ Department of Surgery, University of Cambridge and NIHR Cambridge Biomedical Research Centre, Cambridge, UK. ${ }^{3}$ Wellcome Trust Sanger Institute, Hinxton, UK. ${ }^{4}$ Norwegian PSC Research Center, Division of Cancer, Surgery and Transplantation, Oslo University Hospital, Rikshospitalet, Oslo, Norway. ${ }^{5} \mathrm{~K}$.G. Jebsen Inflammation Research Centre, Research Institute of Internal Medicine, Oslo University Hospital, Rikshospitalet, Oslo, Norway. ${ }^{6}$ Institute of Clinical Medicine, University of Oslo, Oslo, Norway. ${ }^{7}$ Department of Hepatology, Cambridge University Hospitals NHS Foundation Trust, Cambridge, UK. ${ }^{8}$ Department of Histopathology, Cambridge University Hospitals NHS Foundation Trust, Cambridge, UK. ${ }^{9}$ Child Health Clinical Academic Grouping, King's Health Partners, Denmark Hill Campus, London, UK. ${ }^{10}$ Department of Medicine, Division of Gastroenterology and Hepatology, University of Cambridge, Cambridge, UK. ${ }^{11}$ Department of Medicine, School of Clinical Medicine, University of Cambridge, Cambridge, UK. ${ }^{12}$ These authors contributed equally to this work. ${ }^{13}$ These authors share senior authorship for this work. Correspondence should be addressed to L.V. (Iv225@cam.ac.uk).
} 
we adapted our established hepatic hiPSC differentiation protocol ${ }^{11,12}$. Cells generated with the adapted protocol after 12 days of differentiation expressed hepatoblast markers, including $\alpha$-fetoprotein $(A F P)$, HNF4A, HNF1B, TBX3 and CK19 (KRT19) (Fig. 1b,d), and had the potential to differentiate toward both the hepatic (Supplementary Fig. 2a-c) and biliary (Fig. 1c,d and Supplementary Table 1) lineages. To differentiate these hepatoblast-like cells into cholangiocyte progenitors (CPs), we interrogated pathways reported to control early biliary specification ${ }^{10}$ (Supplementary Fig. $3 \mathbf{a}-\mathbf{c}$ and data not shown) and found that activin in combination with retinoic acid suppressed the expression of the hepatoblast markers AFP, HNF4A and TBX3 (Supplementary Fig. 3c). Addition of fibroblast growth factor (FGF)-10 along with activin and retinoic acid induced the expression of the early biliary specification markers SOX 9, HNF $1 B$ and CK19 (Fig. 1c,d $)^{10}$, resulting in a population in which $75.1 \%$ of cells were CK $19^{+}$Sox $^{+}$(Supplementary Fig. 4a). Flow cytometry analyses identified the majority of the remaining cells as Sox9- $\mathrm{AFP}^{+}$hepatoblasts (Supplementary Fig. 4a), explaining the presence of reduced but detectable levels of AFP in our culture (Fig. 1d). Mature biliary markers such as secretin receptor (SCR, also known as SCTR), somatostatin receptor 2 (SSTR2), aquaporin1 (AQP1) and anion exchanger 2 ( $A E 2$, also known as $S L C 4 A 2)$ were not expressed (Fig. 1d). Thus, activin, retinoic acid and FGF10 promote the differentiation of hepatoblast-like cells into early CLCs or CPs in vitro.

To promote maturation of the CPs, we used three-dimensional culture conditions known to induce cholangiocyte maturation through organoid formation ${ }^{7-9}$. CPs grown in these conditions proliferated rapidly, organized

Figure 1 Generation of CPs from hiPSCs. (a) Overview of the protocol used to differentiate hiPSCs to CLCs. DE, definitive endoderm; FP, foregut progenitors; HB, hepatoblasts; HC: hepatocytes; BMP, bone morphogenetic protein; Ly294002 is a phosphatidylinositol3-OH kinase inhibitor; $\mathrm{CDM}$, chemically defined medium; RPMI, Roswell Park Memorial Institute medium; SB, SB-431542; HGF, hepatocyte growth factor; RA, retinoic acid; EGF, epidermal growth factor; FGF, fibroblast growth factor. (b) Immunofluorescence analyses demonstrating the expression of key hepatoblast markers, as indicated, in day 12 hepatoblasts. DAPI, 4',6-diamidino-2-phenylindole dihydrochloride. Scale bars, $100 \mu \mathrm{m}$. (c) Immunofluorescence analyses demonstrating the expression of early biliary markers by immature CPs on day 16 . Scale bars, $100 \mu \mathrm{m}$. (d) Gene expression profile of hiPSC-derived cells at key stages of biliary differentiation and of primary cholangiocytes (PCs). $n=4$ biological replicates for each stage of differentiation. $n=3$ independent samples for PCs. Center line, median; box, interquartile range (IQR); whiskers, range (minimum to maximum). IQR is not calculated for PCs, as $n=3$. Asterisks represent statistical significance of differences between HBs, CPs and CLCs; \# indicates lack of statistical significance $(P>0.05)$ (one-way ANOVA with Tukey correction for multiple comparisons). Values are relative to the housekeeping gene porphobilinogen deaminase ( $P B G D$, also known as $H M B S$ ).

a b
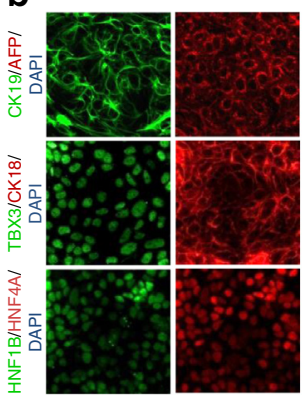

d
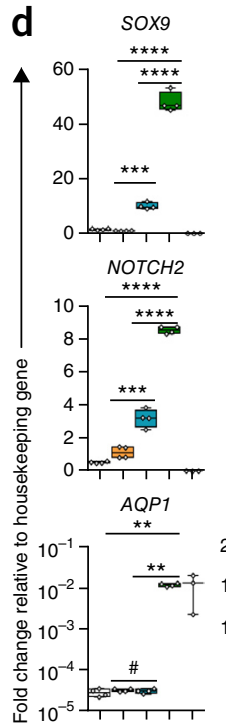

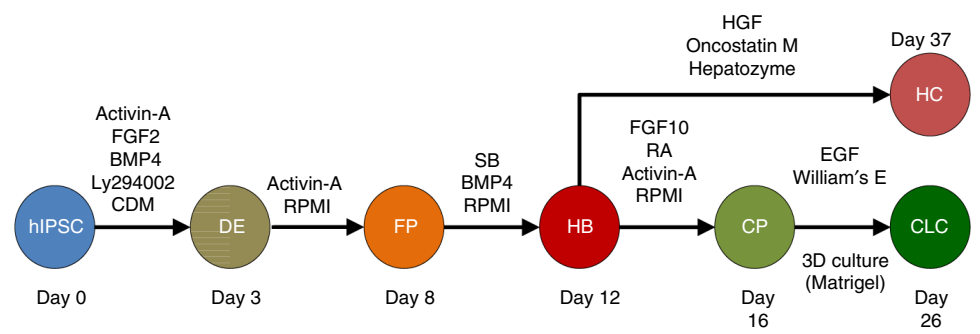

into ring-like structures after $48-72 \mathrm{~h}$ and within $5-7 \mathrm{~d}$ gave rise to cystic organoids and branching tubular structures (Fig. 2a,b) bearing primary cilia (Fig. 2c) similar to those of primary cholangiocytes. The organoids expressed biliary markers, including CK7 (KRT7), CK18 (KRT18), CK19, HNF1B, $\gamma$-glutamyl transferase (GGT1), Jagged1 (JAG1), NOTCH2, CFTR, SCR, SSTR2, AQP1 and AE2 (Figs. 1d and 2d) at levels similar to those in primary cholangiocytes (Fig. 1d and Supplementary Fig. 5). Transcriptomic analyses of common bile duct primary tissue and cells at key stages of our differentiation protocol (Fig. 2e and Supplementary Table 2) using Euclidean hierarchical clustering revealed that CLCs are distinct from earlier developmental stages (Fig. 2e and Supplementary Fig. 6), cluster closely with primary common bile duct cholangiocytes (Pearson correlation coefficient for CLCs versus primary cholangiocytes $r=0.747$, CLCs versus hepatoblasts $r=0.576$, CLCs versus hiPSCs: $r=0.474)$ and express both mature (SSTR2, ALPL, KRT7) and fetal (SOX9) biliary markers, as shown by immunofluorescence and quantitative PCR (QPCR) analyses (Fig. 2e and Supplementary Fig. 6). These results confirm that CPs can differentiate into cells resembling biliary epithelial cells when grown in three-dimensional culture. 
The efficiency of our differentiation protocol can be estimated from the observation that, by day $26,1 \times 10^{6} \mathrm{hiPSC}$ produced $74.4 \times 10^{6}$ cells, of which $57.28 \times 10^{6}$ expressed mature biliary markers $\left(74.4 \times 10^{6}\right.$ cells generated $\times 77 \%$ (average differentiation efficiency across three lines, s.d. $=6.5 \%)=57.28 \times 10^{6}$ CLCs) (Supplementary Fig. 4a,b). More specifically, $74.5 \%$ of the resulting cells expressed both the biliary marker Sox 9 and the mature biliary marker CK7 (Supplementary Fig. 4a). A further $7.5 \%$ of the cells, consistent with immature cholangiocytes, expressed Sox9 but not CK7 (Supplementary Fig. 4a); 15\% of the cells expressed both AFP and albumin

(Supplementary Fig. 4a), indicating the presence of a small fraction of hepatocytes in our culture conditions and explaining the detection of low AFP levels by QPCR analyses (Fig. 1d). The remaining 3\% of the cells were not characterized further. These results were confirmed on three independent hiPSC lines (Supplementary Fig. 4a).

Next we characterized the functionality of the generated organoids. In vivo, cholangiocytes reabsorb bile acids ${ }^{13}$ and modify the composition of canalicular bile through a series of secretory and resorptive processes $^{14}$ regulated by intracellular calcium signaling ${ }^{15}$. Native biliary epithelial cells have ALP and GGT activity and proliferate in response to stimuli such as vascular endothelial growth factor (VEGF). The secretory potential of CLCs generated in vitro was confirmed using rhodamine 123, a fluorescent substrate for the cholangiocyte

Figure 2 Generation of CLCs from CPs. (a,b) Immunofluorescence (left) and light microscopy (right) images of CLC organoids (day 26) demonstrating the formation of cystic (a) and branching (arrows) tubular structures (b). Scale bars, $100 \mu \mathrm{m}$.

(c) Transmission electron microscopy images (right) and immunofluorescence analyses (left) for acetylated $\alpha$-tubulin demonstrating the presence of cilia (arrowhead). The immunofluorescence image was acquired from the bottom of a large cystic organoid using confocal microscopy. Scale bars, $500 \mathrm{~nm}$ and $100 \mu \mathrm{m}$, respectively. L, lumen.

(d) Immunofluorescence analyses demonstrating the expression of early and mature biliary markers in day $25 \mathrm{CLCs}$ as indicated. Scale bars, $100 \mu \mathrm{m}$. (e) Euclidean hierarchical clustering analysis focusing on the genes that define the transcriptional signature of CLCs and HBs $(4,963$ genes differentially expressed in CLCs versus hiPSCs or in HBs versus hiPSCs). For each probe, standard scores ( $z$-scores) indicate the differential expression measured in number of s.d. from the average across all samples. CLCs cluster closer to and present higher correlation coefficient with common bile duct cholangiocytes (CBD) used as a primary control, rather than to HBs or hiPSCs (Pearson, CLCs versus PCs $r=0.747$, CLCs versus HBs $r=0.576$, CLCs versus hiPSCs $r=0.474$ ). Representative genes are indicated. Data correspond to biological triplicates. a
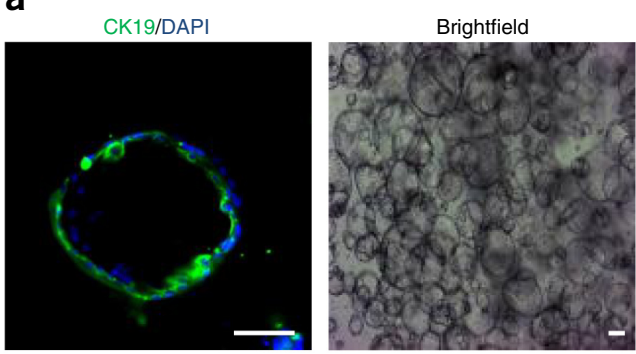

b

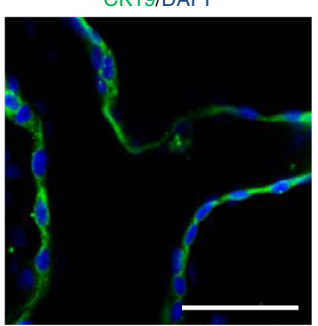

C

Acetylated $\alpha$-tubulin/DAPI

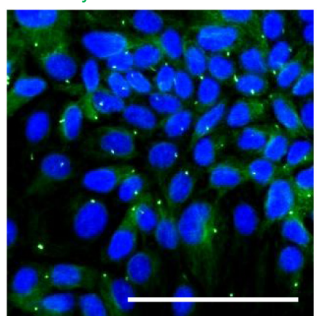

d

CK19/Sox9/ DAPI

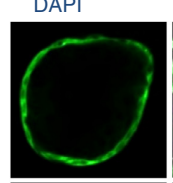
DAPI
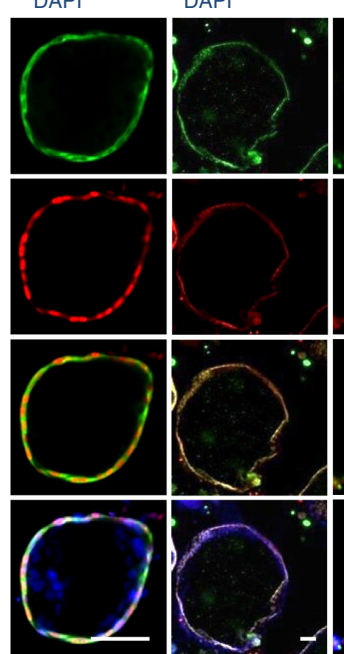

surface glycoprotein multidrug resistance protein-1 (MDR1) ${ }^{16,17}$. Rhodamine 123 was actively secreted in the lumen of CLC organoids, and luminal dye accumulation was prevented by the MDR1 inhibitor verapamil (Fig. 3a-c), confirming MDR1-dependent transfer of rhodamine 123 . The capacity of CLCs to interact with bile acids through the apical salt and bile transporter (ASBT) ${ }^{13}$ was demonstrated by showing active export of the fluorescent bile acid cholyl-lysyl-fluorescein (CLF) from the lumen of CLF-loaded organoids compared to controls loaded with fluorescein isothiocyanate 
(Fig. 3d-f and Supplementary Videos 1 and 2). ASBT expression was confirmed by QPCR and immunofluorescence analyses (Supplementary Fig. 7a,b). Furthermore, CLCs responded to acetylcholine and ATP stimuli by increasing intracellular calcium (Fig. $\mathbf{3 g}$ and Supplementary Videos 3 and 4), demonstrated increased proliferation in response to VEGF stimulation ( $51 \%$ increase in expansion, $P<0.0001$, two-tailed $t$-test) (Fig. 3h,i) and exhibited GGT and ALP activities similar to those of primary controls (GGT activity: $160 \%$ of human serum activity, $P<0.0001$, one-way ANOVA with Dunnett correction for multiple comparisons) (Fig. 3j,k). Together, these observations confirm that our hiPSC-derived CLCs display a range of functions of the native biliary epithelium.

\section{CLCs model development of the human biliary system}

To investigate potential applications of our system for developmental studies, we characterized signaling pathways that control organoid formation in vitro as compared with native duct development. First, we interrogated activin-TGF- $\beta$ signaling in view of its pivotal role in physiological biliary specification and tubulogenesis ${ }^{10,18,19}$. We blocked the activity of TGF- $\beta$, which is normally present in Matrigel, using the activin receptor inhibitor SB-431542. SB-431542 completely abrogated organoid formation (Fig. 4a,b), confirming that activin-TGF- $\beta$ signaling is a key regulator of organoid formation in our system.

We performed similar analyses for Notch signaling during biliary specification of hepatoblasts to CPs and CLC organoid formation in vitro. Deregulation of Notch signaling is associated with Alagille syndrome, characterized by a paucity of bile ducts ${ }^{20}$. To explore the hypothesis that blocking Notch signaling would impair organoid formation in vitro, we first characterized the activity of the Notch pathway in our system. Notch activation results in cleavage and nuclear translocation of its intracellular domain ${ }^{21-23}$. Immunofluorescence analysis with antibodies specific to the cleaved Notch intracellular domain (NICD) confirmed the presence of active NICD, with increased nuclear localization in CPs (Supplementary Fig. 8) and CLC organoids (Fig. 4d). The expression of NOTCH2, as well as its ligand $J A G 1$ and its downstream target HES1, were also increased in both stages compared to hepatoblasts, consistent with pathway activation (CPs versus hepatoblasts: NOTCH2, $P<0.001$; JAG1, $P<0.001$; HES1, $P<0.05$; CLCs versus CPs or hepatoblasts: $P<0.0001$, one-way ANOVA with Tukey correction for multiple comparisons) (Fig. 1d). Inhibition of Notch signaling in three-dimensional culture conditions using the $\gamma$-secretase inhibitor L-685,458 blocked cleavage of the NICD, suppressed HES1, NOTCH2 and JAG1
Figure 3 Functional characterization of CLC organoids. (a) Representative images demonstrating the MDR1 fluorescent substrate rhodamine 123 detected in the lumen of CLC organoids, confirming MDR1 functionality. Scale bars, $50 \mu \mathrm{m}$. (b) Fluorescence intensity measurements along the red line in a. (c) Mean intraluminal fluorescence intensity normalized to background, in the presence (+VER) or absence (-VER) of verapamil; $n=599$ measurements, $P=2.99 \times 10^{-5}$ (two-tailed t-test). (d) Representative images demonstrating active export of the fluorescent bile acid CLF from the lumen of CLC organoids compared to controls loaded with fluorescein isothiocyanate (FITC). Scale bars, $50 \mu \mathrm{m}$. (e) Fluorescence intensity along the red line in $\mathbf{d}$. (f) Mean intraluminal fluorescence intensity normalized to background; $n=1,163$ measurements, $P<1 \times 10^{-18}$ (two-tailed $t$-test). Data in a-f are representative of 3 different experiments. (g) Fluorescence intensity measurements (top) and representative fluorescence microscopy images (bottom) of CLC organoids loaded with the calcium indicator Fluo-4, demonstrating an increase in intracellular calcium upon stimulation with ATP and acetylcholine. Plated primary cholangiocytes stimulated with ATP are used as a positive control. Gray shading represents 1 s.d.; $n=3$. Scale bars, $100 \mu \mathrm{m}$. Boxed areas are magnified in insets. $(\mathbf{h}, \mathbf{i})$ Fold change over starting number of cells ( $h$ ) and immunofluorescence analyses for the proliferation marker $\mathrm{Ki}-67$ (i) in the presence and absence of VEGF for $5 \mathrm{~d}$, demonstrating that VEGF promotes CLC proliferation. Scale bars, $100 \mu \mathrm{m}$. $n=10, P=4.77 \times 10^{-17}$ (CLCs), $P=4.63 \times 10^{-17}$ (plated primary cholangiocytes (prim. chol.)) (two-tailed t-test). (j) CLC organoids exhibit

GGT activity. MEF, mouse embryonic feeders; $n=3, P<0.0001$ for all comparisons (one-way ANOVA with Dunnett correction for multiple comparisons). (k) ALP staining revealing ALP activity in CLC organoids. Top, photographs of stained wells. Scale bars, $1 \mathrm{~cm}$. Bottom, brightfield microscopy images; $n=3$. Boxed area is magnified in inset. Scale bars, $100 \mu \mathrm{m}$. Data representative of 3 lines (Fig. $\mathbf{6 a}, \mathbf{b}$ and Supplementary Fig. 5). All error bars represent s.d. Asterisks $\left(^{* * *}\right)$ in panels $\mathbf{c}, \mathbf{f}, \mathbf{h}$ and $\mathbf{j}$ indicate statistical significance of the differences demonstrated $(P<0.0001)$.
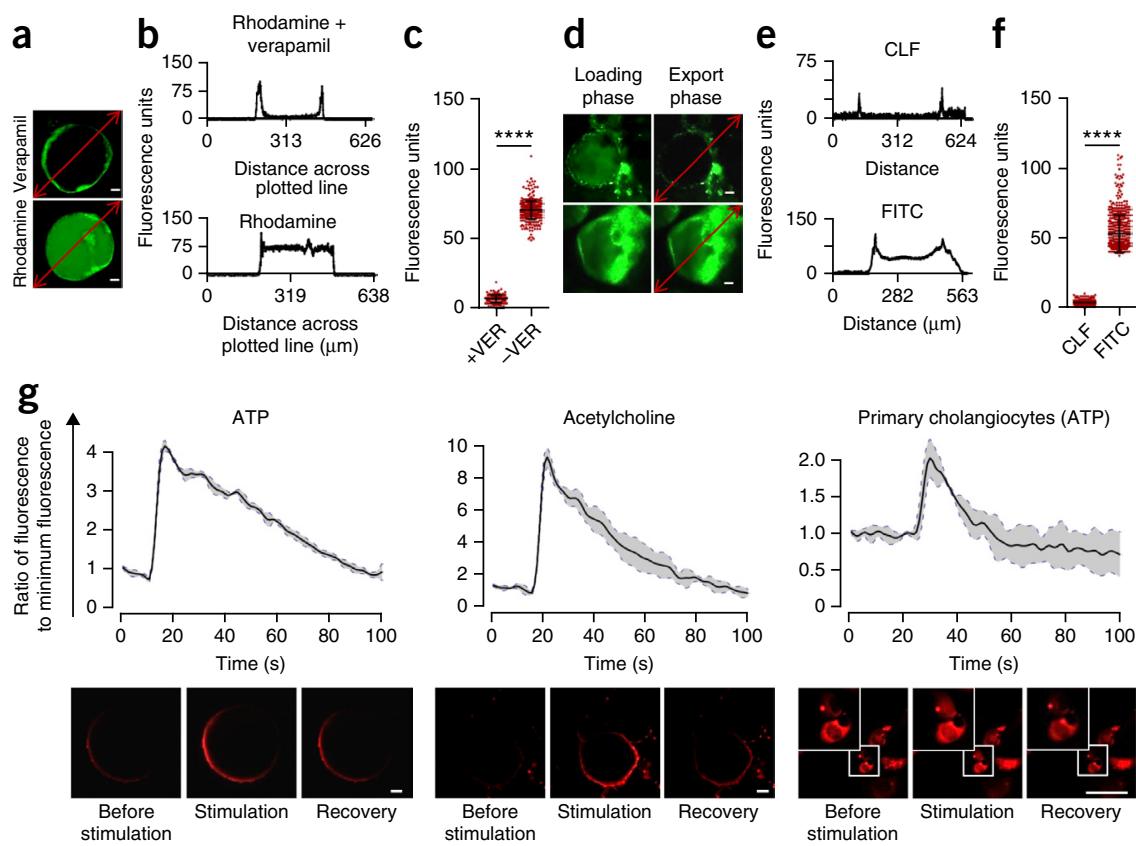

h

\section{i}
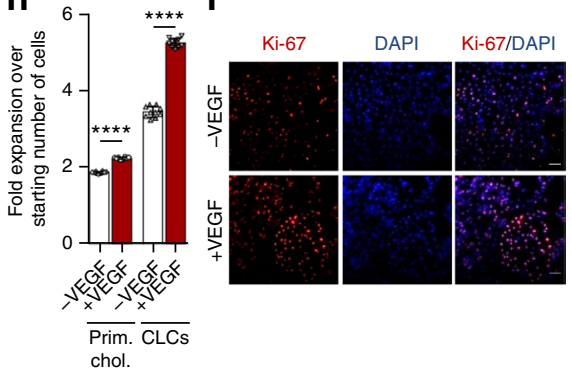

i
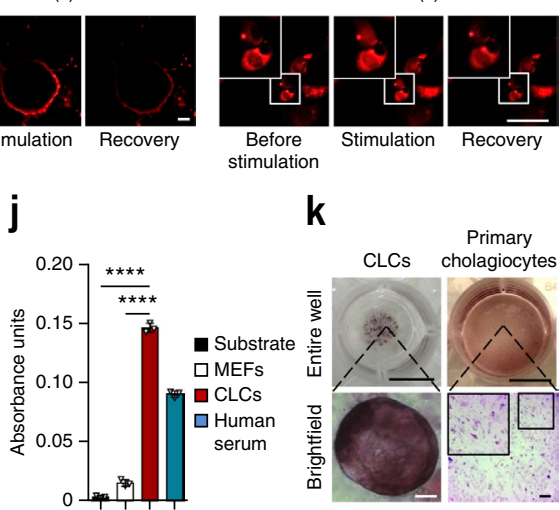

k

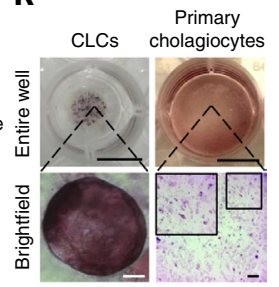


expression (Fig. 4c) and blocked organoid formation (Fig. 4e,f), confirming the importance of Notch signaling to the generation of organoids incorporating a luminal space in our system.

These results reinforce previous findings obtained in mice by demonstrating the importance of activin-TGF- $\beta$ and Notch signaling pathways in human cholangiocyte specification ${ }^{24}$. They underline the potential of our culture system for studying human biliary tree development in vitro.

\section{CLCs validate drugs for polycystic liver diseases}

Polycystic liver diseases are characterized by multiple cystic lesions in the liver arising from fetal cholangiocytes ${ }^{25,26}$. Intraluminal fluid secretion and cholangiocyte proliferation result in cyst expansion and liver impairment owing to space-occupying effects ${ }^{27,28}$. We explored the use of CLC organoids to identify compounds that might reduce cyst size in polycystic liver disease. The secretory activity of cholangiocytes is increased by the hormone secretin and reduced by the hormone somatostatin, resulting in changes in duct size. Octreotide, a synthetic analog of somatostatin, is used clinically to restrict cyst size in polycystic liver disease ${ }^{28-32}$. CLC organoids express both SCR and SSTR2 (Figs. 1d and 5a,b), suggesting that these pathways may be functional in our cells. Accordingly, secretin increased organoid size (6.1\% average diameter increase, $P<0.01$, one-way ANOVA with Dunnett correction for multiple comparisons), whereas somatostatin and octreotide decreased organoid size, compared to untreated controls ( $7.9 \%$ and $4.9 \%$ average diameter decrease, respectively; $P<0.001$ and $P<0.05$, respectively; one-way ANOVA with Dunnett correction for multiple comparisons) (Fig. 5c,d and Supplementary Videos 5-7).
Furthermore, octreotide negated the effects of secretin and decreased intracellular cyclic AMP (45\% of somatostatin response; $P=0.001$, one-way ANOVA with Dunnett correction for multiple comparisons), in keeping with previous studies ${ }^{26,30}$ (Fig. 5e). To further test the effects of octreotide on disease-specific CLCs, we differentiated hiPSCs derived from a patient with polycystic liver disease (Supplementary Fig. 9) to CLCs (Fig. 5f). Octreotide treatment reduced organoid size $(4.86 \%, P<0.0001$, one-way ANOVA with Dunnett correction for multiple comparisons) (Fig. 5g,h), reproducing the effects of the drug in vitro.

\section{CLCs model CF liver disease}

The autosomal recessive disorder CF is caused by mutations in the CFTR gene, encoding a cell-surface chloride transporter ${ }^{33,34}$. CFTR mutations in cholangiocytes result in reduced intraluminal chloride secretion, increased bile viscosity and focal biliary cirrhosis secondary to bile plugs occluding the intrahepatic bile ducts ${ }^{35-37}$. To model CF biliary disease in vitro, we generated hiPSCs from skin fibroblasts of a patient homozygous for the most common CF mutation, $\Delta F 508$ $(\mathrm{CF}-\mathrm{hiPSCs})^{38}$, and differentiated them into CLCs. CF-hiPSC-derived CLCs (CF-CLCs) expressed markers (Fig. 6a) and displayed functional characteristic (Fig. 6b) of biliary epithelial cells. Transcription of the CFTR gene was confirmed using QPCR (Fig. 6a), and immunofluorescence analyses detected minimal CFTR protein expression (Fig. 6c), in agreement with studies reporting very rapid endoplasmic reticulum degradation of the misfolded protein ${ }^{39}$. We also used the fluorescent chloride indicator $N$-(6-methoxyquinolyl)acetoethyl ester (MQAE) ${ }^{40}$ to monitor intracellular and intraluminal chloride
Figure 4 Activin and Notch signaling are essential for CLC organoid formation. (a) Number of CLC organoids following culture of CPs in Matrigel in the presence and absence of SB-431542, demonstrating suppression of organoid formation upon inhibition of activin signaling. Error bars represent s.d., $n=4$. (b) Live cultures demonstrating lack of organoid formation in response to SB-431542. Scale bars, $100 \mu \mathrm{m}$. (c) QPCR analyses for the expression JAG1, NOTCH2 and the Notch downstream target HES1 in CLC organoids versus CPs cultured in Matrigel in the presence of L-685,458, demonstrating reduced expression of this marker in response to $\mathrm{L}-685,458, n=4$. Values are relative to the housekeeping gene $P B G D$ ). Center line, median; box, interquartile range (IQR); whiskers, range (minimum to maximum). ${ }^{* * * *} P<0.0001$ (two-tailed $t$-test). (d) Immunofluorescence analyses for NICD in CLC organoids versus CPs cultured in Matrigel in the presence of L-685,458, demonstrating pathway activation and nuclear localization of NICD in CLC organoids and pathway inhibition in response to L-685,458. Scale bars, $100 \mu \mathrm{m}$; EL, extraluminal space. (e) Number of CLC organoids following culture of CPs in Matrigel in the presence and absence of L-685,458, demonstrating a reduction in organoid formation following inhibition of Notch signaling. Error bars represent s.d.; $n=4$. (f) Live cultures demonstrating reduced organoid formation in response to L-685,458. Scale bars, $100 \mu \mathrm{m}$. Data in each panel are representative of 3 different experiments. a

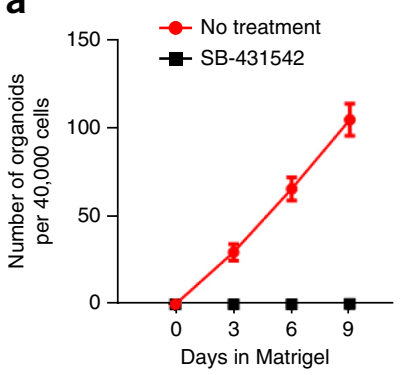

b SB-431542

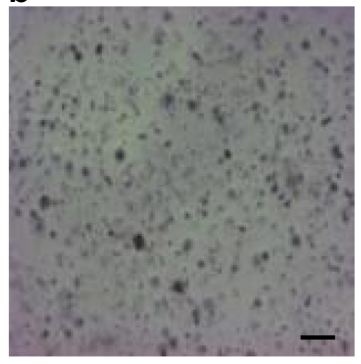

C

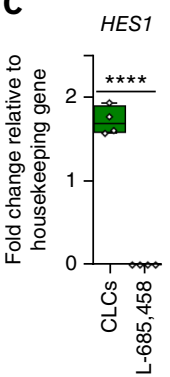

d

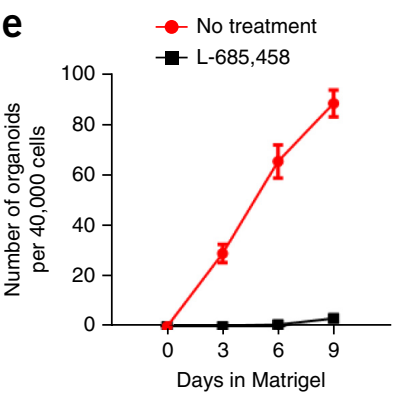
JAG1
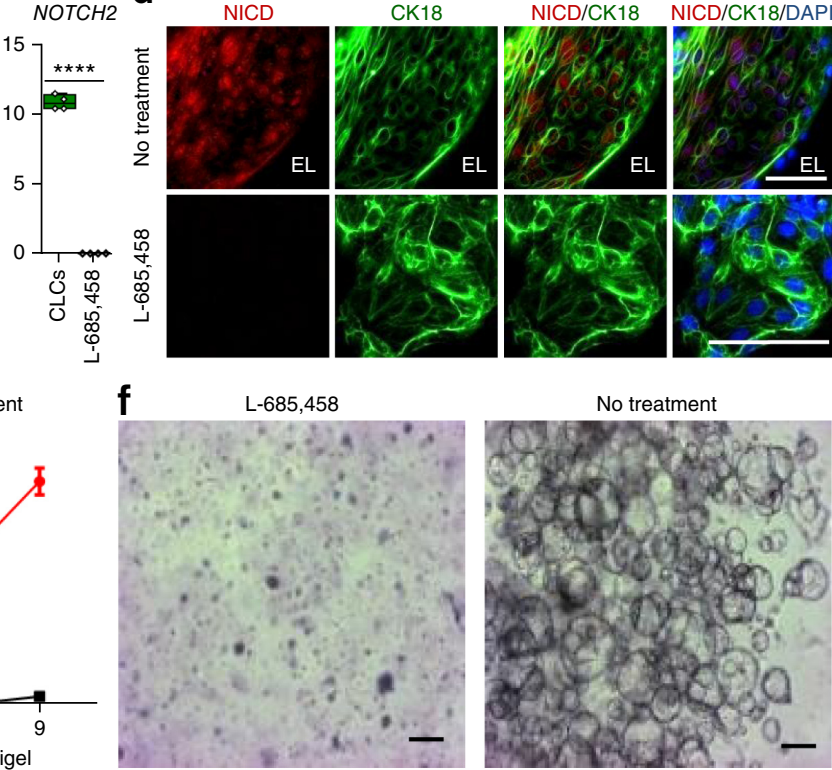
concentration. Wild-type (WT) CLC organoids appropriately modified intracellular chloride in response to media with varying chloride concentrations, whereas no change was observed in CF-CLCs (Fig. 6d,e), confirming the absence of functional CFTR in these cells.
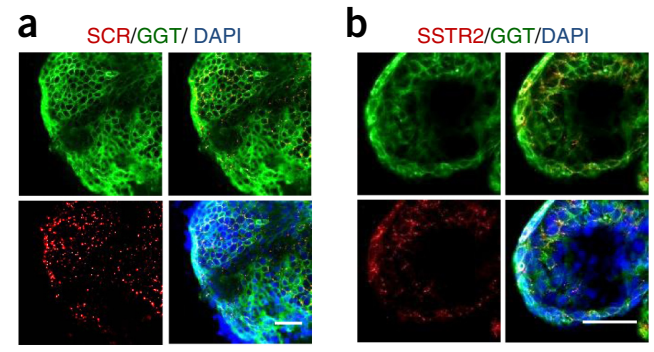

d

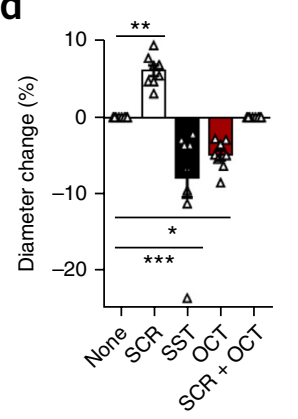

e
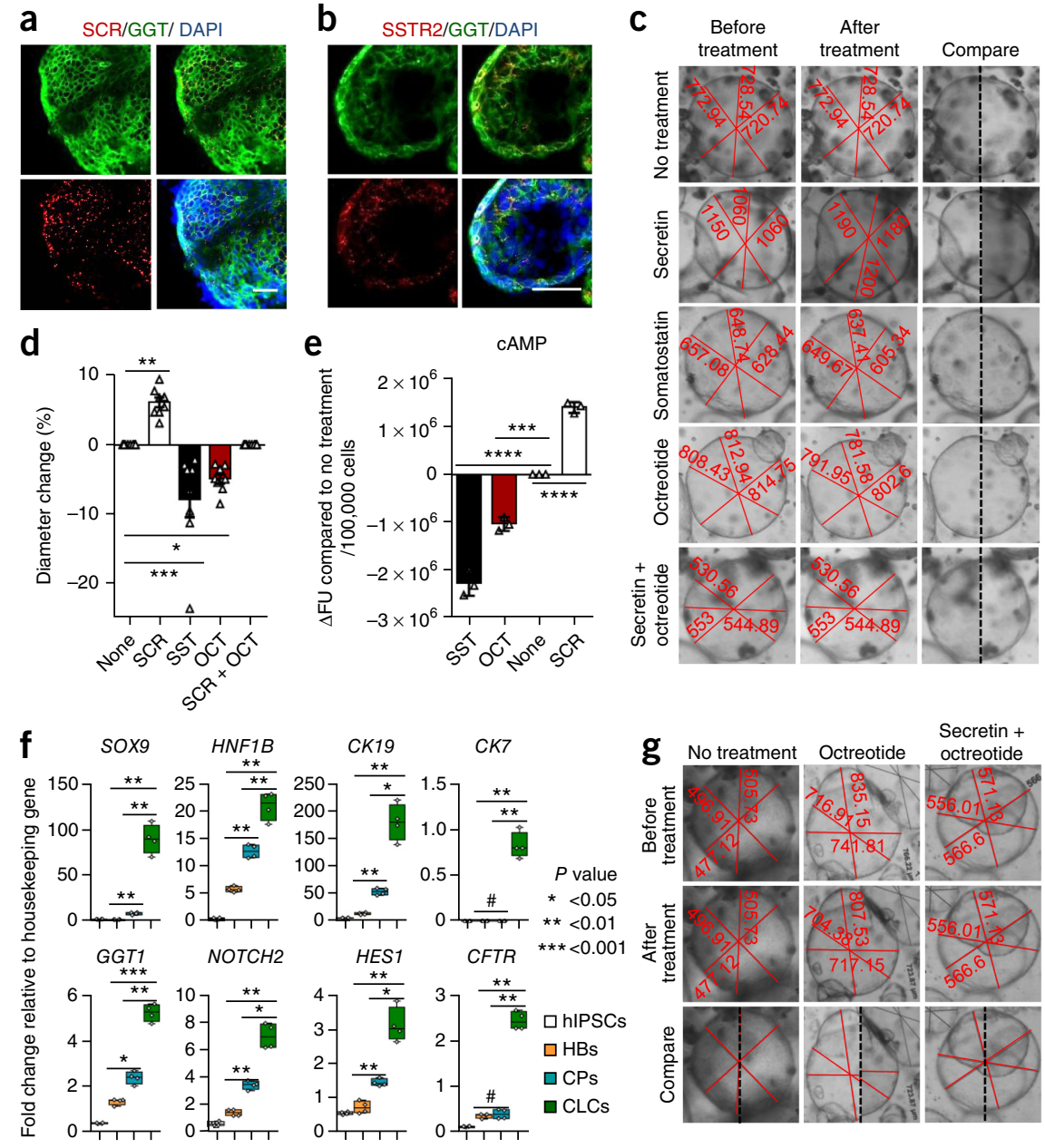

Figure $5 \mathrm{CLC}$ organoids respond to secretin and somatostatin stimuli and reproduce the effects of somatostatin analog (octreotide) in polycystic liver disease in vitro. (a) Immunofluorescence analysis demonstrating the expression of secretin receptor (SCR) in CLCs. Scale bar, $100 \mu \mathrm{m}$. (b) Immunofluorescence analysis demonstrating the expression of somatostatin receptor 2 (SSTR2) in CLCs. Scale bar, $100 \mu \mathrm{m}$. (c) Live cultures demonstrating CLC organoids before and after treatment with secretin, somatostatin, octreotide or secretin combined with octreotide. Diameter measurements in micrometers are shown. The images have been cropped to include a single cyst, but are representative of all cysts measured. (d) Effect of secretin (SCR), somatostatin (SST), octreotide (OCT) and the combination of secretin and octreotide on organoid diameter. Error bars represent s.e.m.; $n=8$, ${ }^{*} P<0.05$, ${ }^{* *} P<0.01,{ }^{* * *} P<0.001$ (one-way ANOVA with Dunnett correction for multiple comparisons). (e) Secretin treatment increases, while somatostatin and octreotide treatment decrease, cAMP in CLC organoids. $\triangle \mathrm{FU}$, change in fluorescence units. Error bars represent s.e.m.; $n=3 ;{ }^{* *} P<0.001$, $* * * * P<0.0001$ (one-way ANOVA with Dunnett correction for multiple comparisons). (f) QPCR demonstrating the expression of biliary markers in polycystic liver disease CLCs. Values are relative to the housekeeping gene PBGD. Center line, median; box, interquartile range (IQR); whiskers, range (minimum to maximum). Asterisks represent statistical significance in differences between HBs, CPs and CLCs; \# indicates lack of statistical significance $(P>0.05)$ (one-way ANOVA with Tukey correction for multiple comparisons). (g,h) Live images (g) and diameter measurements (h) in polycystic liver disease CLC organoids before and after treatment with octreotide or the combination of secretin and octreotide; ${ }^{* * * *} P<0.0001$ (one-way ANOVA with Dunnett correction for multiple comparisons). Error bars represent s.e.m.; $n=8$. Data are representative of 3 different experiments.
Next we investigated the effects of the experimental CF drug VX809 (ref. 41) in the context of biliary disease. VX809 stabilizes CFTR, corrects folding defects in patients with the $\Delta F 508$ mutation and increases CFTR functionality in lung cells ${ }^{42}$. Incubation of CF-CLCs with VX809 for $48 \mathrm{~h}$ increased CFTR function analyzed by MQAE to a level similar to that of WT-CLCs (Fig. 6d,e). This effect was negated by CFTR inhibitor 172, confirming that the phenotypic rescue of CF-CLCs by VX809 depended on improved CFTR function (Fig. 6d,e).

Given the association between chloride and fluid secretion in cholangiocytes ${ }^{33}$, we studied the impact of VX809 on organoid size. CF-CLC organoids treated with VX809 increased in size compared to their untreated counterparts (5.6\% mean diameter increase, $P=0.001$, two-tailed $t$-test) (Fig. 6f,g). This observation confirmed that VX809 increases CFTR function and improves intraluminal fluid secretion, suggesting a previously unreported therapeutic effect for this drug in the context of CF liver disease.

\section{DISCUSSION}

We present a protocol for the generation of CLCs from hiPSCs that addresses the limitations of previously published differentiation methods by more closely recapitulating natural bile duct development (Fig. 1a). Our results confirm the importance of FGF10 and activin-TGF- $\beta$ for early biliary specification, as previously described in vivo ${ }^{10,19,43}$, and reveal a role for retinoic acid in this process, at least in vitro. We show that the combination of these factors under chemically defined, serum-free conditions is sufficient to promote the specification of hiPSC-derived hepatoblasts into CPs with high efficiency. Production of CPs, mimicking physiological biliary development, has not been demonstrated previously, to our knowledge, and likely explains the efficiency of our culture system in generating CLCs that closely resemble bona fide cholangiocytes at the transcriptional (Supplementary Fig. 1 and Supplementary Tables 3 and 4 ) and functional (Supplementary Table 3) levels.

Our protocol will enable various applications, such as developmental studies, disease modeling, therapeutic target validation and drug screening. Accordingly, we used patient-derived hiPSCs to model polycystic and CF liver disease and applied these models to reproduce the effects of the therapeutic compounds verapamil, octreotide and VX809. VX809 has already completed phase 2a clinical trials ${ }^{42}$ for CF, but its effects on CF-associated cholangiopathy have not been described, to our knowledge. Our results suggest that CLCs provide a suitable system for identifying new therapeutic agents, which is particularly 
Figure 6 Modeling CF liver disease in vitro using hiPSCs derived from patients with CF. (a) QPCR analyses of $\mathrm{CLC}$ organoids generated from CF-hiPSCs (CF-CLC), demonstrating the expression of biliary markers. Asterisks denote statistical significance in differences between HBs, CPs and CLCs (one-way ANOVA with Tukey correction for multiple comparisons). Values are relative to the housekeeping gene $P B G D$. Center line, median; box, interquartile range (IQR); whiskers, range (minimum to maximum). (b) CF-CLC organoids exhibit GGT activity. $* * * * P<0.0001$ (one-way ANOVA with Dunnett correction for multiple comparisons). Error bars represent s.d. (c) Immunofluorescence analyses, revealing much lower CFTR protein expression in CF-CLC than in WT-CLCs expressing CFTR. Scale bars, $100 \mu \mathrm{m}$. (d) MQAE fluorescence intensity, normalized to the lowest intensity value. MQAE fluorescence is quenched in the presence of chloride but not affected by nitrate. Changes in intracellular or intraluminal chloride in response to extracellular chloride changes depend on the presence of CFTR functionality. MQAE fluorescence increases in response to a nitrate challenge depleting extracellular chloride and decreases in response to chloride in WT- and CF-CLCs treated with VX809, but fails to respond to both challenges in CF-CLCs and CF-CLCs treated with VX809 plus CFTR inhibitor 172. Error bars represent s.d. (e) Live images of CLC organoids demonstrating an increase in MQAE fluorescence in response to a nitrate challenge, followed by a decrease in response to a chloride challenge, in WT-CLCs and CF-CLCs treated with VX809, whereas MQAE fluorescence remains unchanged in CF-CLCs and CF-CLCs treated with VX809 and CFTR inhibitor 172. Scale bars, $100 \mu \mathrm{m}$. (f) Live images of CLC organoids before and after treatment with VX809. Diameter measurements in micrometers are shown. (g) Effect of VX809 treatment on mean organoid diameter. Error bars represent s.d.; $n=8$, $* * * P=0.001$ (two-tailed $t$-test). All images are cropped to include one cyst, but are representative. All data are representative of 3 different experiments.
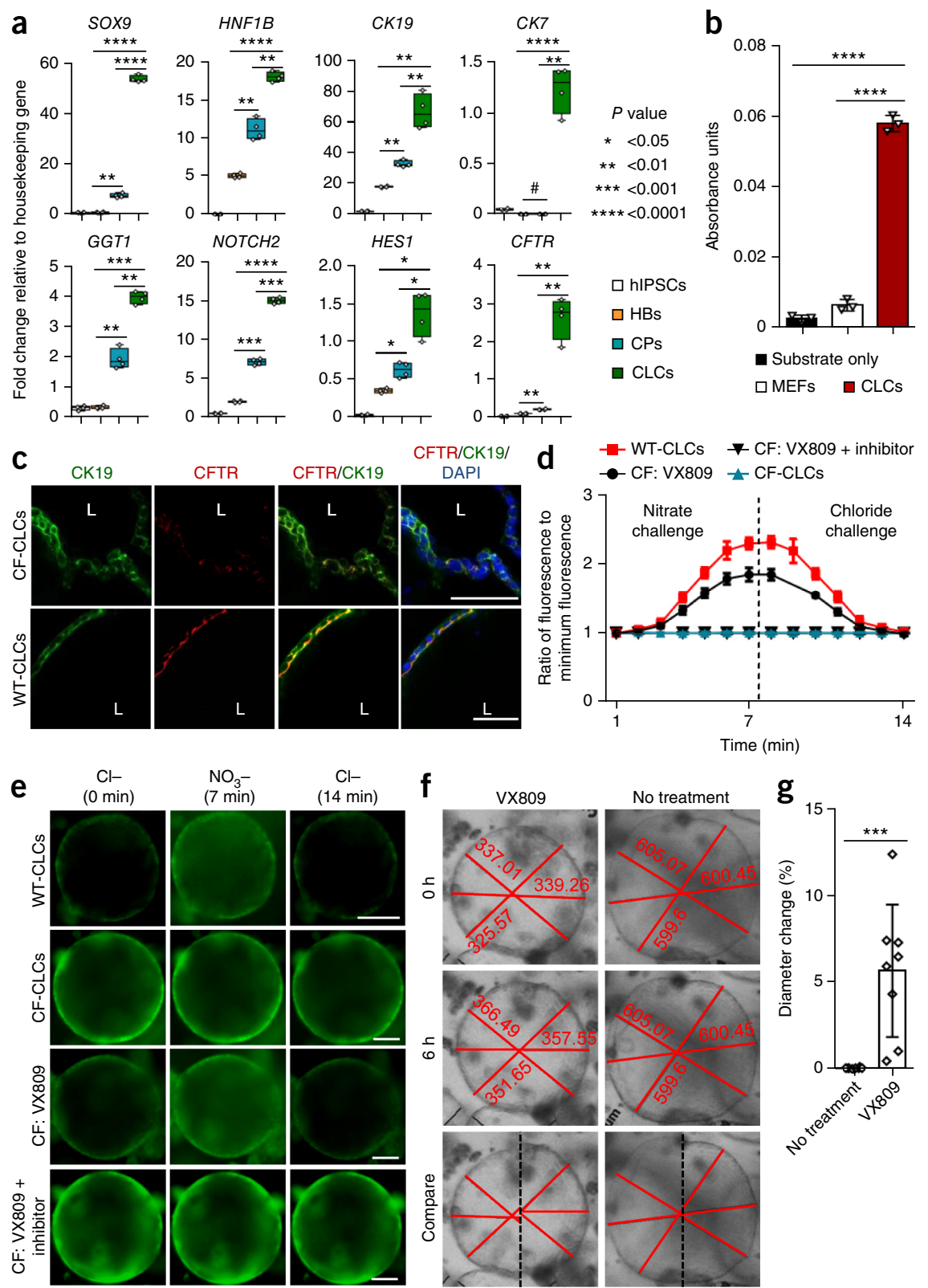

important given the lack of high-throughput drug screening platforms for cholangiopathies. CLCs may also contribute to tissue engineering of livers or liver organoids that incorporate a biliary system for the treatment of end-stage disease.

\section{METHODS}

Methods and any associated references are available in the online version of the paper.

\section{Accession codes. ArrayExpress: E-MTAB-2965.}

Note: Any Supplementary Information and Source Data files are available in the
online version of the paper. ACKNOWLEDGMENTS

The authors would like to thank the Cambridge BRC hiPSCs core facility for the derivation of the cystic fibrosis hiPSC line, P.-A. Tsagkaraki for her help with the generation of the manuscript figures and statistical analyses, J. Skepper, L. Carter and the University of Cambridge Advanced Imaging Centre for their help with electron microscopy, C. McGee and the Wellcome Trust Sanger Institute for their help with microarray data processing and analysis, B. McLeod for IT support and S. Brown for technical support and advice. This work was funded by European Research Council starting grant Relieve IMDs (L.V., N.H.), the Cambridge Hospitals National Institute for Health Research Biomedical Research Center (L.V., N.H., F.S.), the Evelyn Trust (N.H.), the European Union FP7 grant TissuGEN (M.C.d.B.) and core support grant from the Wellcome Trust and UK Medical Research Council to the Wellcome Trust - Medical Research Council Cambridge Stem Cell Institute. F.S. has been supported by an Addenbrooke's Charitable Trust Clinical Research Training Fellowship and a joint UK Medical Research CouncilSparks Clinical Research Training Fellowship.

\section{AUTHOR CONTRIBUTIONS}

F.S.: design and concept of study, execution of experiments and data acquisition, development of protocols and validation, generation, collection and interpretation of data, production of figures, manuscript writing, editing and final approval of manuscript. M.C.d.B., F.A.C.S.: technical support, execution of experiments. P.M.: bioinformatics and statistical analyses. A. Bertero: bioinformatics analyses. K.S.-P., E.S., E.M.: primary tissue provision. T.H.K., J.A.B., W.T.H.G., S.D., A. Baker, A.K., G.J.A.: critical revision of the manuscript for important intellectual content. N.R.F.H.: design and concept of study, study supervision, generation 
and interpretation of data, editing and final approval of manuscript. L.V.: design and concept of study, study supervision, interpretation of data, editing and final approval of manuscript.

\section{COMPETING FINANCIAL INTERESTS}

The authors declare competing financial interests: details are available in the online version of the paper.

Reprints and permissions information is available online at http://www.nature.com/ reprints/index.html.

1. Lazaridis, K.N., Strazzabosco, M. \& Larusso, N.F. The cholangiopathies: disorders of biliary epithelia. Gastroenterology 127, 1565-1577 (2004).

2. Murray, K.F., Carithers, R.L. Jr. \& AASLD. AASLD practice guidelines: evaluation of the patient for liver transplantation. Hepatology 41, 1407-1432 (2005).

3. Pollheimer, M.J., Trauner, M. \& Fickert, P. Will we ever model PSC? - "it's hard to be a PSC model!". Clin. Res. Hepatol. Gastroenterol. 35, 792-804 (2011).

4. Takahashi, K. \& Yamanaka, S. Induction of pluripotent stem cells from mouse embryonic and adult fibroblast cultures by defined factors. Cell 126, 663-676 (2006).

5. Robinton, D.A. \& Daley, G.Q. The promise of induced pluripotent stem cells in research and therapy. Nature 481, 295-305 (2012).

6. Sampaziotis, F., Segeritz, C.P. \& Vallier, L. Potential of human induced pluripotent stem cells in studies of liver disease. Hepatology doi:10.1002/hep.27651 (2014).

7. Zhao, D. et al. Derivation and characterization of hepatic progenitor cells from human embryonic stem cells. PLoS ONE 4, e6468 (2009).

8. Tanimizu, N., Miyajima, A. \& Mostov, K.E. Liver progenitor cells develop cholangiocyte-type epithelial polarity in three-dimensional culture. Mol. Biol. Cell 18, 1472-1479 (2007).

9. Dianat, N. et al. Generation of functional cholangiocyte-like cells from human pluripotent stem cells and HepaRG cells. Hepatology 60, 700-714 (2014).

10. Si-Tayeb, K., Lemaigre, F.P. \& Duncan, S.A. Organogenesis and development of the liver. Dev. Cell 18, 175-189 (2010).

11. Rashid, S.T. et al. Modeling inherited metabolic disorders of the liver using human induced pluripotent stem cells. J. Clin. Invest. 120, 3127-3136 (2010).

12. Hannan, N.R., Segeritz, C.P., Touboul, T. \& Vallier, L. Production of hepatocyte-like cells from human pluripotent stem cells. Nat. Protoc. 8, 430-437 (2013).

13. Xia, X., Francis, H., Glaser, S., Alpini, G. \& LeSage, G. Bile acid interactions with cholangiocytes. World J. Gastroenterol. 12, 3553-3563 (2006).

14. Kanno, N., LeSage, G., Glaser, S., Alvaro, D. \& Alpini, G. Functional heterogeneity of the intrahepatic biliary epithelium. Hepatology 31, 555-561 (2000).

15. Minagawa, N., Ehrlich, B.E. \& Nathanson, M.H. Calcium signaling in cholangiocytes. World J. Gastroenterol. 12, 3466-3470 (2006).

16. Gigliozzi, A. et al. Molecular identification and functional characterization of Mdrla in rat cholangiocytes. Gastroenterology 119, 1113-1122 (2000).

17. Cízková, D., Morký, J., Micuda, S., Osterreicher, J. \& Martínková, J. Expression of MRP2 and MDR1 transporters and other hepatic markers in rat and human liver and in WRL 68 cell line. Physiol. Res. 54, 419-428 (2005).

18. Antoniou, A. et al. Intrahepatic bile ducts develop according to a new mode of tubulogenesis regulated by the transcription factor SOX9. Gastroenterology 136 , 2325-2333 (2009).

19. Clotman, F. et al. Control of liver cell fate decision by a gradient of TGF beta signaling modulated by Onecut transcription factors. Genes Dev. 19, 1849-1854 (2005).

20. Turnpenny, P.D. \& Ellard, S. Alagille syndrome: pathogenesis, diagnosis and management. Eur. J. Hum. Genet. 20, 251-257 (2012).
21. Bray, S.J. Notch signalling: a simple pathway becomes complex. Nat. Rev. Mol. Cell Biol. 7, 678-689 (2006).

22. Saravanamuthu, S.S., Gao, C.Y. \& Zelenka, P.S. Notch signaling is required for lateral induction of Jagged1 during FGF-induced lens fiber differentiation. Dev. Biol. 332, 166-176 (2009)

23. Geisler, F. \& Strazzabosco, M. Emerging roles of Notch signaling in liver disease. Hepatology 61, 382-392 (2015).

24. Zong, Y. et al. Notch signaling controls liver development by regulating biliary differentiation. Development 136, 1727-1739 (2009).

25. Raynaud, P. et al. A classification of ductal plate malformations based on distinct pathogenic mechanisms of biliary dysmorphogenesis. Hepatology 53, 1959-1966 (2011).

26. Chandok, N. Polycystic liver disease: a clinical review. Ann. Hepatol. 11, 819-826 (2012).

27. Temmerman, F. et al. Systematic review: the pathophysiology and management of polycystic liver disease. Aliment. Pharmacol. Ther. 34, 702-713 (2011).

28. Caroli, A. et al. Reducing polycystic liver volume in ADPKD: effects of somatostatin analogue octreotide. Clin. J. Am. Soc. Nephrol. 5, 783-789 (2010).

29. Marinelli, R.A. et al. Secretin induces the apical insertion of aquaporin-1 water channels in rat cholangiocytes. Am. J. Physiol. 276, G280-G286 (1999).

30. Gong, A.Y. et al. Somatostatin stimulates ductal bile absorption and inhibits ducta bile secretion in mice via SSTR2 on cholangiocytes. Am. J. Physiol. Cell Physiol. 284, C1205-C1214 (2003).

31. Caperna, T.J., Blomberg, L.A., Garrett, W.M. \& Talbot, N.C. Culture of porcine hepatocytes or bile duct epithelial cells by inductive serum-free media. In Vitro Cell. Dev. Biol. Anim. 47, 218-233 (2011).

32. Masyuk, T.V., Masyuk, A.I., Torres, V.E., Harris, P.C. \& Larusso, N.F. Octreotide inhibits hepatic cystogenesis in a rodent model of polycystic liver disease by reducing cholangiocyte adenosine 3',5'-cyclic monophosphate. Gastroenterology 132, 1104-1116 (2007).

33. Rowe, S.M., Miller, S.B. \& Sorscher, E.J. Cystic Fibrosis. N. Engl. J. Med. 352 1992-2001 (2005).

34. Davies, J.C., Alton, E.W. \& Bush, A. Cystic fibrosis. Br. Med. J. 335, 1255-1259 (2007).

35. Colombo, C. Liver disease in cystic fibrosis. Curr. Opin. Pulm. Med. 13, 529-536 (2007).

36. Staufer, K., Halilbasic, E., Trauner, M. \& Kazemi-Shirazi, L. Cystic fibrosis related liver disease-another black box in hepatology. Int. J. Mol. Sci. 15, 13529-13549 (2014).

37. Haack, A., Aragão, G.G. \& Novaes, M.R. Pathophysiology of cystic fibrosis and drugs used in associated digestive tract diseases. World J. Gastroenterol. 19, 8552-8561 (2013).

38. Hannan, N.R., Sampaziotis, F., Segeritz, C., Hanley, N. \& Vallier, L. Generation of distal airway epithelium from multipotent human foregut stem cells. Stem Cells Dev. doi:10.1089/scd.2014.0512 (2015).

39. Ward, C.L. \& Kopito, R.R. Intracellular turnover of cystic fibrosis transmembrane conductance regulator. Inefficient processing and rapid degradation of wild-type and mutant proteins. J. Biol. Chem. 269, 25710-25718 (1994).

40. Shenoy, A. et al. Calcium-modulated chloride pathways contribute to chloride flux in murine cystic fibrosis-affected macrophages. Pediatr. Res. 70, 447-452 (2011).

41. Van Goor, F. et al. Correction of the F508del-CFTR protein processing defect in vitro by the investigational drug VX-809. Proc. Natl. Acad. Sci. USA 108, 18843-18848 (2011)

42. Clancy, J.P. et al. Results of a phase Ila study of VX-809, an investigational CFTR corrector compound, in subjects with cystic fibrosis homozygous for the F508delCFTR mutation. Thorax 67, 12-18 (2012).

43. Yanai, M. et al. FGF signaling segregates biliary cell-lineage from chick hepatoblasts cooperatively with BMP4 and ECM components in vitro. Dev. Dyn. 237, 1268-1283 (2008). 


\section{ONLINE METHODS}

Generation of hiPSC lines. All hiPSC lines used here were derived and characterized previously by our lab ${ }^{11,37}$. Briefly, the lines used were generated from human skin fibroblasts and peripheral blood (ethics reference no. 08/H0311/201 for Hertfordshire Regional Ethics Committee (REC) and 09/H0304/77 for National Research Ethics Service (NRES) Committee East of England, Cambridge East), using the Yamanaka approach ${ }^{4,9}$. The CF fibroblasts were obtained from the Coriell cell repository. The lines were authenticated using SNIP arrays and regularly tested negative for mycoplasma contamination.

Culture of hiPSCs. Human iPS cells were maintained in defined culture conditions as previously described ${ }^{11,12,44}$, using activin-A $(10 \mathrm{ng} / \mathrm{ml})$ and b-FGF (12 ng/ml).

Differentiation of hiPSCs into cholangiocyte progenitors. hiPSCs were differentiated into foregut progenitor cells (FPs) as previously described ${ }^{12,44}$. Bipotent hepatoblasts were generated by culturing FPs in RPMI (Gibco, Invitrogen) plus B27 supplemented with SB-431542 (10 $\mu \mathrm{M}$, Tocris Bioscience) and BMP4 $(50 \mathrm{ng} / \mathrm{ml})$ for $4 \mathrm{~d}$. To induce biliary specification, we cultured hepatoblasts for another $4 \mathrm{~d}$ in the presence of RPMI (Gibco, Invitrogen) plus B27 supplemented with FGF10 (50 ng/ml, Peprotech), activin-A (50 ng/ml) and retinoic acid ( $3 \mu \mathrm{M}$, Sigma-Aldrich).

Maturation of cholangiocyte progenitor cells to CLCs and organoid formation in 3D culture. Human CPs were passaged using Cell Dissociation Buffer (Gibco, Life Technologies, catalog number 13151-014) and suspended at a density of $8 \times 10^{4}$ cells $/ \mathrm{ml}$, in a mixture of $40 \%$ Matrigel (BD Biosciences, catalog number 356237) and 60\% William's E medium (Gibco, Life Technologies) supplemented with $10 \mathrm{mM}$ nicotinamide (Sigma-Aldrich), $17 \mathrm{mM}$ sodium bicarbonate (Sigma-Aldrich), $0.2 \mathrm{mM}$ 2-phospho-L-ascorbic acid trisodium salt (Sigma-Aldrich), $6.3 \mathrm{mM}$ sodium pyruvate (Invitrogen), $14 \mathrm{mM}$ glucose (Sigma-Aldrich), 20 mM HEPES (Invitrogen), ITS+ premix (BD Biosciences), $0.1 \mu \mathrm{M}$ dexamethasone (R\&D Systems), $2 \mathrm{mM}$ Glutamax (Invitrogen), $100 \mathrm{U} / \mathrm{ml}$ penicillin, $100 \mu \mathrm{g} / \mathrm{ml}$ streptomycin and $20 \mathrm{ng} / \mathrm{ml} \mathrm{EGF}$ (R\&D Systems). A 50- $\mu \mathrm{L}$ droplet of the cell suspension was added in the center of each well of a 24-well plate; the gel was allowed $1 \mathrm{~h}$ at $37^{\circ} \mathrm{C}$ to solidify and then overlaid with William's $\mathrm{E}$ medium with supplements. The medium was changed every $48 \mathrm{~h}$ and the cells were cultured for a total of $10 \mathrm{~d}$.

Using the same methodology, we have been able to culture CLC organoids in formats ranging from 6- to 96-well plates. To generate large numbers of CLC organoids, multiple $50-\mu \mathrm{L}$ droplets were added in a well of a 6 -well plate or a $10-\mathrm{cm}$ dish. To provide a large number of wells compatible with high-throughput screening and large-scale experiments, $30-\mu \mathrm{L}$ droplets were added per well of a 96-well plate. In both cases, the gel was allowed $1 \mathrm{~h}$ at $37^{\circ} \mathrm{C}$ to solidify and then overlaid with William’s E medium with supplements.

Inhibition of activin and Notch signaling in $3 \mathrm{D}$ culture and assessment of organoid formation. Human CPs were suspended at a density of $8 \times 10^{4}$ cells $/ \mathrm{ml}$, in a mixture of $40 \%$ Matrigel and $60 \%$ William's E medium (Gibco, Life Technologies) with supplements as described above. The cell suspension was distributed in three aliquots of equal volume. One aliquot received no further supplementation and was used as a positive control. The second aliquot was further supplemented by $10 \mu \mathrm{M} \mathrm{SB}-431542$ for the inhibition of activin-TGF- $\beta$ signaling. $50 \mu \mathrm{M}$ L-685,458 (Tocris Biosciences) was added in the third aliquot for the inhibition of Notch signaling. Each aliquot was distributed in 24-well-plate format. The same concentrations of inhibitors were added to the medium overlaying the Matrigel on a daily basis. After a total of $10 \mathrm{~d}$ in $3 \mathrm{D}$ culture, the total number of cysts in four random wells of a 24-well plate was counted for each condition by a researcher blinded to sample identity. Error bars represent s.d.

Flow cytometry analyses. HiPSCs, FPs, HBs and CPs were dissociated to single cells using Cell Dissociation Buffer (Life Technologies). The cells were then counted using a hemocytometer and fixed using 4\% PFA for $20 \mathrm{~min}$ at $4{ }^{\circ} \mathrm{C}$. Cell staining and flow cytometry analyses were performed as previously described ${ }^{44}$.
CLC organoids were washed once with PBS and $1 \mathrm{ml}$ of ice-cold dispase was added per well of a 24-well plate. The Matrigel was mechanically dissociated, transferred to a Falcon tube and kept on ice to allow the combination of low temperature and dispase digestion to liquefy the Matrigel. After $10 \mathrm{~min}$ the cells were centrifuged at 1,600 r.p.m. (444g) for 3 min and the supernatant was aspirated. The pellet was washed once with PBS and the centrifugation step repeated. The supernatant was aspirated and $1 \mathrm{ml}$ of TrypLE (Life Technologies) was added for 3-5 min until the organoids were dissociated to single cells. Finally, the single cell suspension was centrifuged at 1,600 r.p.m. $\left(13.2 \mathrm{~g}\right.$ ) for $3 \mathrm{~min}$, and fixed using $4 \%$ PFA for $20 \mathrm{~min}$ at $4{ }^{\circ} \mathrm{C}$. Cell staining and flow cytometry analyses were performed as previously described ${ }^{44}$.

Primary cholangiocytes. Frozen primary human cholangiocytes derived from common bile duct were obtained from Celprogen (catalog number 36755-11). The cells were derived from donors negative for hepatitis B surface antigen, HIV 1 and 2, syphilis, hepatitis B virus, human T lymphotropic viruses 1 and 2, hepatitis $\mathrm{C}$ virus, West Nile virus and Trypanosoma cruzi. Each frozen ampule $\left(1.2 \times 10^{6}\right.$ cells $)$ of $1 \times 10^{6}$ viable cells was thawed with gentle agitation in a $37^{\circ} \mathrm{C}$ water bath. The cells were transferred to a sterile centrifuge tube and $1 \mathrm{ml}$ of prewarmed Human Cholangiocyte Cell Culture Complete Growth Medium (Celprogen, catalog number M36755-11S) was added. The ampule was washed twice with Human Cholangiocyte Cell Culture Complete Growth Medium and the cells were centrifuged at $100 \mathrm{~g}$ for $7 \mathrm{~min}$. The pellet was resuspended in $500 \mu \mathrm{l}$ of Human Cholangiocyte Cell Culture Complete Growth Medium. The cells were distributed equally to two wells of a 6-well plate precoated with Human Cholangiocyte Cell Culture Extracellular Matrix Celprogen, catalog number M36755-11-6-wells) with $2 \mathrm{ml}$ of Human Cholangiocyte Cell Culture Complete Growth Medium per well. The cells were incubated at $37^{\circ} \mathrm{C}$ in a $5 \%$ $\mathrm{CO}_{2}$, humidified incubator for $48 \mathrm{~h}$ and the medium was changed once. After another $24 \mathrm{~h}$, when the wells were confluent, the cells were lysed and RNA was extracted as previously described ${ }^{11,12}$, or the cells were fixed with $4 \%$ PFA as previously described ${ }^{11,12}$ for ALP staining. QPCR analyses for biliary markers (Fig. 1d) and ALP staining (Fig. 3k) confirmed the nature and cholangiocyte properties of the cells.

Primary biliary tissue. Primary biliary tissue (bile duct) was obtained from an organ donor from whom organs were being retrieved for transplantation. A section of the bile duct was excised during the multi-organ retrieval operation after obtaining informed consent from the donor's family (REC reference number: 09/H0306/73, NRES Committee East of England-Norfolk). The tissue was homogenized using a tissue homogenizer and RNA was extracted as previously described ${ }^{11}$.

Immunofluorescence, RNA extraction and quantitative real-time PCR. Immunofluorescence, RNA extraction and QPCR were performed as previously described ${ }^{9}$. A complete list of the primary and secondary antibodies used is provided in Supplementary Table 5. A complete list of the primers used is provided in Supplementary Table 6. All QPCR data correspond to four independent biological replicates, with the exception of the primary $\mathrm{CDB}$ cholangiocytes from Celprogen, where three independent samples were used.

For immunofluorescence in 3D Matrigel cultures, the organoids were fixed in Matrigel with 4\% PFA for $20 \mathrm{~min}$ at room temperature, to avoid Matrigel liquefaction. The samples were permeabilized and blocked with $0.1 \%$ Triton $\mathrm{X}-100$ and $10 \%$ donkey serum respectively for $30 \mathrm{~min}$ and incubated with primary antibody in $1 \%$ donkey serum overnight at $4{ }^{\circ} \mathrm{C}$. The following day the samples were washed three times with PBS for $45 \mathrm{~min}$ per wash, incubated with secondary antibody in $1 \%$ donkey serum for $60 \mathrm{~min}$ at room temperature and washed again three times in PBS. Hoechst 33258 was added to the first wash.

All immunofluorescence images were acquired using a Zeiss Axiovert 200M inverted microscope or a Zeiss LSM 700 confocal microscope. Image $1.48 \mathrm{k}$ software (Wayne Rasband, NIHR, USA, http://imagej.nih.gov/ij) was used for image processing. Changes in brightness or contrast during processing were applied equally across the entire image.

For RNA extraction in 3D Matrigel cultures, the organoids were washed once with PBS and $1 \mathrm{ml}$ of ice-cold dispase was added per well of a 24-well plate. 
The Matrigel was mechanically dissociated, transferred to a Falcon tube and kept on ice to allow the combination of low temperature and dispase digestion to liquefy the Matrigel. After $10 \mathrm{~min}$ the cells were centrifuged at 1,600 r.p.m. (444g) for $3 \mathrm{~min}$ and the supernatant was aspirated. The pellet was washed once with PBS and the centrifugation step repeated. Finally, the supernatant was aspirated and $350 \mu \mathrm{L}$ of RNA lysis buffer were added to the pellet. RNA was extracted from the lysate using a kit (GenElute Mammalian Total RNA Miniprep Kit, Sigma-Aldrich, catalog number RTN350) according to the manufacturer's instructions.

Microarrays. $500 \mathrm{ng}$ of total cellular RNA was amplified and purified using the Illumina TotalPrep-96 RNA Amplification kit (Life Technologies) according to the manufacturer's instructions. Three biological replicates for each condition were analyzed. Biotin-labeled cRNA was then normalized to a concentration of $150 \mathrm{ng} / \mu \mathrm{l}$ and $750 \mathrm{ng}$ were hybridized to Illumina Human-12 v4 BeadChips for $16 \mathrm{~h}$ (overnight) at $58{ }^{\circ} \mathrm{C}$. Following hybridization, BeadChips were washed and stained with streptavidin-Cy3 (GE Healthcare). BeadChips were then scanned using the BeadArray reader and image data were processed using Genome Studio software (Illumina). The raw and processed microarray data are available on ArrayExpress (accession code E-MTAB-2965).

Microarray analysis. Probe summaries for all arrays were obtained from the raw data using the method "Making Probe Summary" in Genome Studio (Illumina). These values were transformed (variance stabilized) and quantile normalized using the R/Bioconductor package lumi ${ }^{45}$. Standard lumi QC procedure was applied and no outliers were identified.

Differential expression between pairs of conditions was evaluated using the $\mathrm{R} /$ Bioconductor package limma ${ }^{46}$. A linear model fit was applied, and the top differentially expressed genes were tabulated for each contrast using the method of Benjamini and Hochberg to correct the $P$ values ${ }^{47}$. Probes that failed to fluoresce above background in both conditions were removed. Differentially expressed probes were selected using a cutoff of adjusted $P<0.01$ and absolute fold-change $>2$.

Probes differentially expressed between hiPSCs and CLCs or hiPSCs and HBs (representing the aggregate transcriptional signature of CLCs and HBs) were selected for Euclidean hierarchical clustering using Perseus software (MaxQuant). Standard scores ( $z$-scores) of the $\log _{2}$-normalized probe expression values across the different conditions were calculated and used for this analysis.

Rhodamine 123 transport assay. CLC organoids were incubated with $100 \mu \mathrm{M}$ rhodamine 123 (Sigma-Aldrich) for $5 \mathrm{~min}$ at $37^{\circ} \mathrm{C}$ and then washed with William's E medium three times. Fresh William's E medium with supplements was added following the third wash. The organoids were incubated at $37^{\circ} \mathrm{C}$ for another $40 \mathrm{~min}$. To demonstrate that rhodamine 123 transfer indeed reflected the activity of the membrane channel MultiDrug Resistance Protein 1 (MDR1), CLCs were incubated with $10 \mu \mathrm{M}$ verapamil (Sigma-Aldrich) at $37^{\circ} \mathrm{C}$ for $30 \mathrm{~min}$ and the rhodamine assay was repeated. Following completion of each experiment, images were taken using a confocal microscope. Around 1,000 fluorescence measurements were made between the organoid interior and exterior. Rhodamine 123 fluorescence in the organoid lumen was normalized to the background measured in the surrounding external area. Each experiment was repeated in triplicate. Error bars represent s.d. Mean fluorescence intensity comparisons were performed using a two-sided Student's $t$-test.

Cholyl-lysyl-fluorescein transport assay. CLC organoids were loaded with $5 \mu \mathrm{M}$ cholyl-lysyl-fluorescein (CLF, Corning Incorporated) for $30 \mathrm{~min}$ at $37^{\circ} \mathrm{C}$ and then washed with Leibovitz's medium (Life Technologies) three times. Following completion of the third wash, time-lapse images were taken using a confocal microscope for $10 \mathrm{~min}$. To demonstrate that the changes in CLF fluorescence intensity observed were secondary to active export of CLF from the organoid lumen, the experiment was repeated with $5 \mu \mathrm{M}$ unconjugated fluorescein isothiocyanate (FITC) (Sigma-Aldrich) as a control. Around 1,000 fluorescence measurements were made between the organoid interior and exterior.
Fluorescence in the organoid lumen was normalized to background measured in the surrounding external area. Each experiment was repeated in triplicate. Error bars represent s.d. Mean fluorescence intensity comparisons were performed using a two-sided Student's $t$-test.

Measurement of intracellular calcium. Intracellular calcium signaling, regulated by stimuli such as acetylcholine and ATP, constitutes a key second messenger for cholangiocytes ${ }^{15}$. CLC organoids were incubated with $25 \mu \mathrm{M}$ of the calcium indicator Fluo-4 a.m. (Life Technologies) for $60 \mathrm{~min}$ at $37^{\circ} \mathrm{C}$ and washed three times with William's E medium. Fresh William's E medium with supplements was added following the third wash. The organoids were stimulated with $1 \mu \mathrm{M}$ acetylcholine (Sigma-Aldrich) or $30 \mu \mathrm{M}$ ATP (SigmaAldrich), while time-lapse images were taken. Each measurement was repeated in triplicate. To calculate the number of cells responding to stimulation, the number of cells loaded with Fluo- 4 a.m. was counted by two different researchers before the start of the experiment. Following stimulation with ATP or acetylcholine the number of responding cells (increase in fluorescence) was also counted and responsiveness was expressed as the ratio of responding cells to the total number of cells loaded with Fluo- 4 a.m. For the statistical approach for smoothing the data and plotting bands for the confidence limits, please see "Statistical analyses."

Proliferation assays. Twenty $50-\mu \mathrm{L}$ droplets of Matrigel, each containing 40,000 cells, were distributed in 20 wells of a 24 -well plate. VEGF at a concentration of $50 \mathrm{ng} / \mathrm{ml}$ was added to half of the wells with every medium change. Following $5 \mathrm{~d}$ of culture, the Matrigel was digested with dispase as described in "RNA extraction" above and the organoids were mechanically dissociated to single cells. The number of cells for each well was then counted using a hemocytometer. Twenty different measurements were made by a researcher blinded to sample identity. Primary cholangiocytes distributed in 20 wells of two 12 -well plates were used as a positive control. Ten wells received VEGF at a concentration of $50 \mathrm{ng} / \mathrm{ml}$ with every medium change for $5 \mathrm{~d}$, after which the number of cells in each well was counted as described above. Error bars represent s.d. Mean cell number comparisons were performed using a two-sided Student's $t$-test.

GGT activity. GGT activity was measured in triplicate using the MaxDiscovery gamma-Glutamyl Transferase (GGT) Enzymatic Assay Kit (Bioo Scientific) according to the manufacturer's instructions. Mouse embryonic feeders were used as a negative control. The equivalent serum GGT activity in IU/L was calculated following the manufacturer's instructions by multiplying the average increase in absorbance over 10 min by 353. Error bars represent s.d. Multiple mean absorbance comparisons (CLCs versus substrate, CLCs versus MEFs, CLCs versus human serum) were performed using one-way ANOVA with Dunnett correction for multiple comparisons.

Alkaline phosphatase staining. Alkaline phosphatase staining was carried out using the BCIP/NBT color development substrate (5-bromo-4-chloro3-indolyl-phosphate/nitro blue tetrazolium, Promega) according to the manufacturer's instructions.

Effect of secretin, somatostatin, octreotide and VX809 on organoid size. Images of CLC organoids were taken using $5 \times$ magnification before and after the addition of secretin (100 nM, Sigma-Aldrich), somatostatin (100 nM, Sigma-Aldrich), octreotide (100 nM, Sigma-Aldrich) or embryo transfer water, serving as a negative control, at $0.5-2 \mathrm{~min}$ intervals until organoid size stabilized. To explore the impact of octreotide on the effect of secretin, cells were preincubated for $30 \mathrm{~min}$ with octreotide. $100 \mathrm{nM}$ secretin (SigmaAldrich) was subsequently added to the medium and the experiment was carried out as described above. To assess the effect of VX809 on organoid size, images were taken before and $6 \mathrm{~h}$ after the addition of VX809 (30 mM, Selleck) or embryo transfer water, serving as a negative control. Three random diameters were measured for eight random organoids before and after treatment. Graph measurements represent percentage differences in mean organoid diameter. Error bars represent s.d. Statistical significance was calculated using one-way ANOVA with Dunnett correction for multiple comparisons. 
Supplementary Videos 5-7 were made by taking images before and after treatment at 2-min intervals until organoid size stabilized.

cAMP levels. cAMP levels were measured in triplicate using the cAMP-Glo assay kit (Promega) according to the manufacturer's instructions and a P450Glomax 96 microplate luminometer (Promega). Error bars represent s.d. Statistical significance was calculated using one-way ANOVA with Dunnett correction for multiple comparisons.

CFTR activity. CFTR activity was measured as previously described ${ }^{37}$. Briefly, MQAE is a fluorescent dye quenched by the presence of chloride but not affected by other anions or $\mathrm{pH}$ changes ${ }^{39}$. Chloride transfer across the cell membrane is mainly regulated by CFTR in cholangiocytes. Therefore, cells with a functional CFTR will respond to a chloride challenge by rapidly increasing intracellular (and intraluminal, in the case of organoids) chloride concentration, thereby quenching MQAE fluorescence. Chloride depletion using a nitrate solution will have the opposite effect.

Cells were incubated with $8 \mathrm{mM}$ MQAE (Life Technologies) and $5 \mu \mathrm{M}$ forskolin for $4 \mathrm{~h}$ at $37^{\circ} \mathrm{C}$. Standard Ringer's solution containing $\mathrm{NaCl}, \mathrm{KCl}$, $\mathrm{CaCl}_{2}, \mathrm{MgCl}_{2}$ glucose and HEPES was used to provide a chloride challenge expected to increase intracellular chloride in the presence of functional CFTR. Modified Ringer's solution consisting of $\mathrm{NaNO}_{3}, \mathrm{KNO}_{3}, \mathrm{CaNO}_{3}$, $\mathrm{MgNO}_{3}$, glucose and HEPES was used to promote chloride efflux and deplete intracellular chloride. Live pictures were captured every minute as each solution was added. To demonstrate the effect of VX809 on CFTR functionality, CLC organoids were incubated with $30 \mathrm{mM}$ VX809 (Selleck) for $48 \mathrm{~h}$. The assay was repeated as described above in the presence and absence of $7 \mu \mathrm{M}$ CFTR inhibitor 172 (Sigma-Aldrich) to confirm the specificity of the compound for CFTR. Intracellular fluorescence intensity was measured in three random areas from the wall of each organoid using ImageJ software and normalized to the minimum fluorescence value for each area. Error bars represent s.d.

Cytochrome p450 activity. Cyp3A4 activity was measured using the p450Glo assay kit (Promega) according to the manufacturer's instructions and a P450-Glomax 96 microplate luminometer (Promega).
Timing of experiments on CLC organoids. All the experiments and characterization with regards to CLCs were performed on CLC organoids after $10 \mathrm{~d}$ of $3 \mathrm{D}$ culture unless stated otherwise.

Statistical analyses. All statistical analyses were performed using GraphPad Prism 6 or the R statistical environment. For comparison between two mean values, a two-sided Student's $t$-test was used to calculate statistical significance. For comparison between multiple values, one-way ANOVA was used with Tukey correction for multiple comparisons when comparing multiple values to each other (for example, QPCR plots) or Dunnett correction for multiple comparisons when comparing multiple values to a single value (for example, functional assays where the values are compared to a negative control). The normal distribution of our values was confirmed using the KolmogorovSmirnov test where appropriate. Further information on the statistical analysis of our data is provided in Supplementary Table 7 (test used for each experiment or analysis, test statistic, degrees of freedom, $P$ value).

To smooth our data for generating the curves in Figure 3g, we used functional data analysis theor ${ }^{48}$ implemented in the R package "fda" (http://cran. r-project.org/web/packages/fda/index.html). First, we represented our data values ( 3 replications at each fluorescence intensity measurement) using 60 equidistant $\mathrm{B}$-spline basis functions, and roughness penalties in the second derivative $(\lambda=1)$. We used the functions create.bspline.basis and smooth.fd over the interval 1-100 s. Then we evaluated the mean and the s.d. of the functional data objects using the R functions mean.fd and sd.fd.

44. Hannan, N.R. et al. Generation of multipotent foregut stem cells from human pluripotent stem cells. Stem Cell Reports 1, 293-306 (2013).

45. Du, P. \& Kibbe, W. Lin SM lumi: a pipeline for processing Illumina microarray. Bioinformatics 24, 1547-1548 (2008).

46. Smyth, G.K. Linear models and empirical Bayes methods for assessing differential expression in microarray experiments. Stat. Appl. Genet. Mol. Biol. 3, 3 (2004).

47. Benjamini, Y. \& Hochberg, Y. Controlling the false discovery rate: a practical and powerful approach to multiple testing. J. R. Stat. Soc. 57, 289-300 (1995).

48. Ramsay, J.O. \& Silverman, B.W. Functional Data Analysis 2nd edn. (Springer, New York, 2006). 